Chuangchuang Duan

Institute of Mechanics,

Chinese Academy of Sciences,

Beijing 100190, China;

School of Engineering Sciences,

University of Chinese Academy of Sciences,

Beijing 100049, China

e-mail: duanchuangchuang@imech.ac.cn

Yujie Wei ${ }^{1}$

LNM,

Institute of Mechanics,

Chinese Academy of Sciences,

Beijing 100190, China;

School of Engineering Sciences,

University of Chinese Academy of Sciences,

Beijing 100049, China

e-mail: yujie_wei@Inm.imech.ac.cn

\section{Theory on Bending in Cantilever Beams With Adsorbed Islands}

Traction between adsorbed islands and the substrate is commonly seen in both living and material systems: deposited material gathers into islands at the early stage of polycrystalline film deposition and generates stress due to lattice mismatch, cells exert cellular traction to extracellular matrix to probe their surrounding microenvironment in vivo, and so on. The traction between these islands and the substrate can result in perceivable macroscopic deformation in the substrate and may be measurable if the substrate is a cantilever beam. However, currently broadly used Stoney equation is incapable of handling such boundary condition. In this paper, we give the closed-form expression on the resulted curvature in substrate beams by distributed tractions. Such a relationship could be employed to monitor the stress evolution during thin film deposition, to quantify the stress level of cell traction as cells adhere to cantilever beams, and other related mechanical systems like charging-discharging induced stress in island-patterned electrode films. Moreover, we found that follower traction induced by an array of islands could lead to negative curvature. It shields light on the early stage compressive stress during polycrystalline film deposition. [DOI: 10.1115/1.4036819]

Keywords: adsorbed islands, Stoney equation, film deposition, traction, bending

\section{Introduction}

With enhanced capability in microfabrication, there are growing systems made of pattern islands on thin film substrates. Indeed, during Volmer-Weber growth of films, adsorbed atoms gather in distributed and isolated islands at the early stage. Such islands are grain nucleus; their coalescence with neighbors and further growth lead to the final rather flat film [1,2]. Due to lattice mismatch, there exists traction between those islands and film substrate, which results in bending of the substrate $[3,4]$. The film stress is related with the curvature of the substrate by Stoney equation [5]. The stress in the film could be monitored via the multibeam optical stress sensor (MOSS) system and used as a quality control parameter for film deposition [6,7]. Typical experimental observation of stress revolution over the course of deposition shows three-stage process: initial compressive stress during island growth, rapid increase in tensile stress in the film during island coalescence, and finally, a steady stress state in fully postcoalescence. Various studies have been conducted to explain this three-stage process $[8,9]$. For instance, Cammarata et al. proposed a surface stress model to describe the origin of compressive stress prior to coalescence [10]. Bower and coworkers preformed finite element calculation to investigate the stress evolution during the growth of polycrystalline films and developed corresponding theoretical models to capture the stress evolution during different stages of deposition [11-13]. To analysis the origin of thin film stress and relate it to the feature and morphology of the growth surface, Chason et al. grew patterned arrays of islands and measured the stress evolution [14,15].

Similar strategy is employed to battery stress control, a growing field with enormous attention from different disciplines. During charge and discharge, the significant volume change, taking lithium-ion battery as an example, is responsible for the performance of the battery. Different from characterizing the mechanical properties of materials, the mechanics of a battery in service is hard to probe. Guduru and coworkers adopted the MOSS system and measured the evolution of stress during charge-discharge circles of a thin film battery for the first time [16]. Pharr et al.

\footnotetext{
${ }^{1}$ Corresponding author.

Contributed by the Applied Mechanics Division of ASME for publication in the Journal OF APpled Mechanics. Manuscript received April 14, 2017; final manuscript received May 20, 2017; published online May 31, 2017. Assoc. Editor: Kyung-Suk Kim.
}

[17], Bucci et al. [18], and Sethuraman et al. [19] also used this technology to characterize other mechanical properties such as the biaxial modulus, the flow stress, and fracture energy of thin film electrodes. Furthermore, to avoid premature film and minimizing the stress by volume expansion, island-patterned film battery was designed [20,21]. There are many related mechanical analyses related to the failure of such film batteries [22-24]. A closed-form relationship between island stress and film bending curvature is however missing.

Another typical example is the traction between cell and extracellular matrix. It mediates fundamental cell process, including cell morphogenesis, focal adhesions, differentiation, and migration [25-28]. To have a better understanding of how cellular traction impacts these process, various methods have been suggested to measure it over the past decades. The original idea to culture cells on elastic material to extract cellular traction from the deformation of the substrate comes from Harris et al. [29]. Nevertheless, it is a nonlinear process to identify cellular traction from the degree of wrinkle of the substrate. This problem was subsequently solved by using substrate that deformed but not buckled in response to cellular traction [30]. Afterward, traction force microscopy was developed to give a more accurate quantification of cellular traction [31]. With fluorescent beads embedded into the substrate, the displacement field can be easily visualized and recorded. Then, it is convenient to solve the inverse problem of elasticity theory to calculate cellular traction. Another approach to determine cellular traction is the use of multiple deformable posts [32]. Cells are attached to arrays of closely spaced vertical posts and bend the posts like vertical cantilevers, which gives a linear relationship between traction forces and the deflection of posts. With these strategies developed, the traction exerted by one cell can be determined conveniently. In general, the cellular traction within one cell was found to increase with the distance from the cell center [33-35]. A systematic study by Rape et al. showed that cellular traction correlates linearly with the distance from the center of the cell [28].

In this paper, we aim to construct the relationship between the curvature of a cantilever beam and the tractions introduced by patterned islands or the averaged stress introduced by many cells residing on a substrate. We start from solving the elastic problem with one island sitting on substrate beam and then generalize the solution to the case of substrate with multi-islands, as illustrated in Fig. 1. The closed-form relationship between the magnitude of the traction and the curvature of the beam is supplied. 


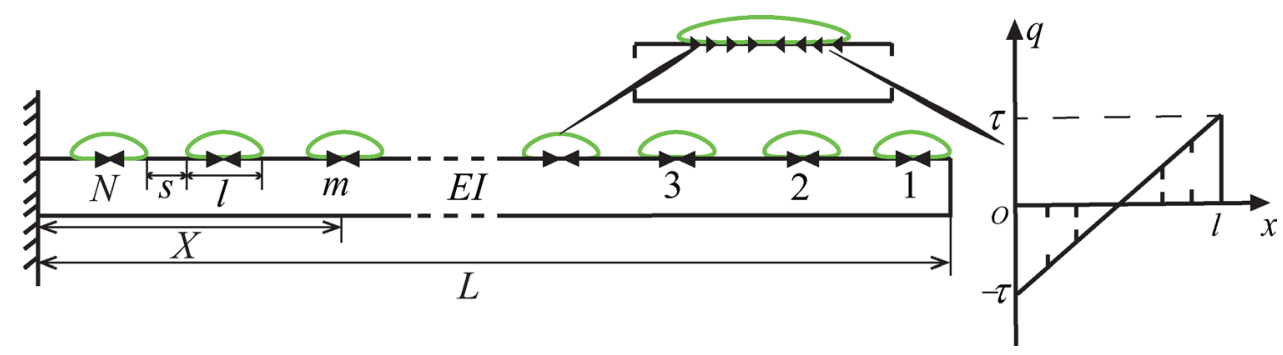

Fig. 1 A cantilever beam with an array of islands bonded to its upper surface, where the lengths of the beam and one island are $L$ and $I$, respectively. $N$ denotes the number of islands and $s$ denotes the length of the spacing between islands. $\tau$ refers to the maximum of the traction and $E I$ refers to the flexural rigidity of the beam.

(a)

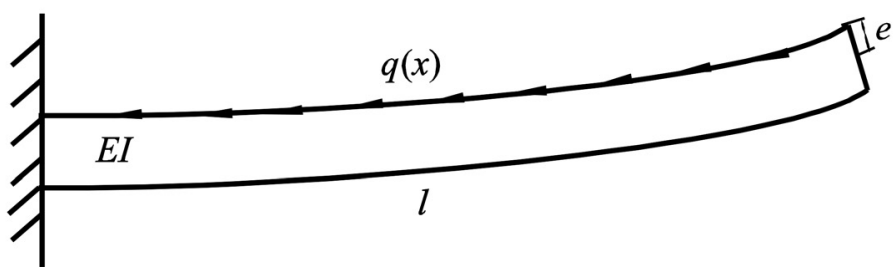

Fig. 2 (a) A cantilever beam subjected to tangential force $f(x)$, where the length of the beam is $I$ and the eccentricity of the tangential force is $e$. (b) An element cut from the beam with sides normal to the deflected axis of the beam.

\section{Modeling and Analytical Solutions}

For the purpose of validity and simplicity, we consider a cantilever beam subjected to periodic distributed traction on its upper surface and the distribution of traction in each of the islands varies linearly from one side of the island to the other, as shown in Fig. 1 .

2.1 Governing Equation of a Single Island. To drive the basic equations, we consider a cantilever beam subjected to arbitrarily distributed tangential follower force as shown in Fig. 2(a). The relations among traction force $f(x)$, axial force $N$,shearing force $Q$, and bending moment $M$ can be obtained from the equilibrium of the element in Fig. 2(b)

Summing forces in the $\eta$ direction gives

$$
\frac{d Q}{d x}=-N \frac{d \theta}{d x}
$$

Taking moments about point $O_{1}$, we obtain

$$
\frac{d M}{d x}=Q-q(x) e
$$

According to the classical beam theory [36], we have

$$
\begin{gathered}
\frac{d \theta}{d x}=\kappa \\
E I \kappa=M
\end{gathered}
$$

Differentiating Eq. (2) with respect to $x$ and combining with Eqs. (1), (3), and (4), we can express the differential equation of the beam in terms of curvature $\kappa(x)$ as

$$
E I \kappa^{\prime \prime}(x)+N \kappa(x)+q^{\prime}(x) e=0
$$

Note that $q(x)=\tau(2 x-l) / l$ and $N=\int_{x}^{l} q(\xi) d \xi$, the governing equation can now be formulated as

$$
\kappa^{\prime \prime}(x)+\frac{\tau}{E I l} x(l-x) \kappa(x)+\frac{2 \tau e}{E I l}=0
$$

The relevant boundary condition for a cantilever beam in Fig. 2 can be obtained from Eqs. (2) and (4)

$$
\kappa(l)=0 \quad \text { and } \quad \kappa^{\prime}(l)=-\frac{\tau e}{E I}
$$

2.2 Analytical Solutions. We now seek a solution of Eq. (6) in the form [37]

$$
\kappa(x)=\sum_{n=0}^{\infty} a_{n}(l-x)^{n}
$$

Given the power series in Eq. (8), we have

$$
\begin{aligned}
2 a_{2} & +\frac{2 \tau e}{E I l}+\left(6 a_{3}+\frac{\tau}{E I} a_{0}\right)(l-x)+\sum_{n=0}^{\infty}\left[(n+4)(n+3) a_{n+4}\right. \\
& \left.+\frac{\tau}{E I} a_{n+1}-\frac{\tau}{E I l} a_{n}\right](l-x)^{n+2}=0
\end{aligned}
$$

For Eq. (9) to be satisfied, the coefficient of each power of $l-x$ must be zero. From the first and second terms, we obtain

$$
a_{2}=-\frac{\tau e}{E I l} \quad \text { and } \quad a_{3}=-\frac{\tau}{6 E I} a_{0}
$$

The third term in Eq. (9) gives the recurrence relation

$$
a_{n+4}=\frac{\tau}{E I l} \frac{1}{(n+4)(n+3)}\left(a_{n}-l a_{n+1}\right), \quad \text { with } \quad n=0,1,2 .
$$

Substituting the boundary condition in Eq. (7) into Eq. (8), we obtain 


$$
a_{0}=0, \quad a_{1}=\frac{\tau e}{E I}
$$

Now, with the recurrence formula in Eq. (11) and the first four coefficients, we are able to determine all the coefficients in Eq. (8), i.e., the solution of Eq. (6) with boundary condition in Eq. (7).

2.3 Multi-Islands. Now, we consider a cantilever beam subjected to multitraction. Observing that the governing equation of each island is the same as written in Eq. (6), the difference between each island lies in the boundary conditions. Hence, Eqs. (10) and (11) are still valid and then all the coefficients $a_{3}, a_{4}$, etc., depend on only two constants, $a_{0}$ and $a_{1}$, which can be determined by using the right-hand boundary condition of each island. We introduce the dimensionless parameters $\bar{x}=x / l$ and $\bar{\tau}=$ $\tau l^{3} / E I$ and rewrite Eq. (8) in terms of $a_{0}$ and $a_{1}$

$$
\begin{aligned}
\kappa(x)= & a_{0} \sum_{n=0}^{\infty} A_{n}(1-\bar{x})^{n}+a_{1} l \sum_{n=0}^{\infty} B_{n}(1-\bar{x})^{n} \\
& -\frac{\tau e l}{E I} \sum_{n=0}^{\infty} C_{n}(1-\bar{x})^{n}
\end{aligned}
$$

where

$$
\begin{aligned}
& A_{0}=1, \quad A_{1}=0, \quad A_{2}=0, \quad A_{3}=-\bar{\tau} / 6, \quad A_{n+4}=\frac{\bar{\tau}}{(n+4)(n+3)}\left(A_{n}-A_{n+1}\right) \\
& B_{0}=0, \quad B_{1}=1, \quad B_{2}=0, \quad B_{3}=0, \quad B_{n+4}=\frac{\bar{\tau}}{(n+4)(n+3)}\left(B_{n}-B_{n+1}\right) \\
& C_{0}=0, \quad C_{1}=0, \quad C_{2}=1, \quad C_{3}=0, \quad C_{n+4}=\frac{\bar{\tau}}{(n+4)(n+3)}\left(C_{n}-C_{n+1}\right)
\end{aligned}
$$

As shown in Figs. 1 and 3, we use the superscript to refer to the sort of the islands with "1" denoting the first island by the free end of the cantilever beam and the superscripts " 0 " and $l$ refer to the left-hand and right-hand boundaries of the island, respectively. From Eqs. (2) and (4), we obtain the boundary conditions of the $m$ th island labeled in Fig. 1 as

$$
\begin{aligned}
M_{l}^{m} & =E I \kappa^{m}(l)=E I a_{0} \\
Q_{l}^{m} & =E I \kappa^{m \prime}(l)+\tau e=-E I a_{1}+\tau e \\
M_{0}{ }^{m} & =E I \kappa^{m}(0) \\
Q_{0}{ }^{m} & =E I \kappa^{m \prime}(0)-\tau e
\end{aligned}
$$

From the first two equations in Eq. (14), we can use $M_{l}{ }^{m}$ and $Q_{l}{ }^{m}$ instead of $a_{0}$ and $a_{1}$ in Eq. (13) and neglect higher order terms of $\bar{\tau}$

$$
\begin{aligned}
k^{m}(x)= & \frac{M_{l}^{m}}{E I}\left[1+\bar{\tau}\left[-\frac{(1-\bar{x})^{3}}{6}+\frac{(1-\bar{x})^{4}}{12}\right]\right]-\frac{Q_{l}^{m} l}{E I}\left[(1-\bar{x})+\bar{\tau}\left[-\frac{(1-\bar{x})^{4}}{12}+\frac{(1-\bar{x})^{5}}{20}\right]\right] \\
& +\frac{\tau e l}{E I}\left[(1-\bar{x})-(1-\bar{x})^{2}+\bar{\tau}\left[-\frac{(1-\bar{x})^{4}}{12}+\frac{(1-\bar{x})^{5}}{10}-\frac{(1-\bar{x})^{6}}{30}\right]\right]
\end{aligned}
$$

Substituting Eq. (15) into the last two equations in Eq. (14) leads to

$$
\begin{aligned}
M_{0}{ }^{m} & =\left(1-\frac{1}{12} \bar{\tau}\right) M_{l}{ }^{m}-\left(1-\frac{1}{30} \bar{\tau}\right) Q_{l}{ }^{m} l-\frac{\bar{\tau}}{60} \tau e l \\
Q_{0}{ }^{m} & =\frac{\bar{\tau}}{6} \frac{M_{l}^{m}}{l}+\left(1-\frac{1}{12} \bar{\tau}\right) Q_{l}{ }^{m}+\frac{\bar{\tau}}{30} \tau e
\end{aligned}
$$

Considering the equilibrium of the spacing in Fig. 3(a), we have

$$
\begin{aligned}
Q_{l}^{m+1} & =Q_{0}^{m} \\
M_{l}^{m+1} & =M_{0}^{m}-s * Q_{0}^{m}
\end{aligned}
$$

Combining Eq. (16) with Eq. (17), we have the recurrence formulation between $Q_{l}^{m+1}, M_{l}^{m+1}$ and $Q_{l}^{m}, M_{l}^{m}$ (a)

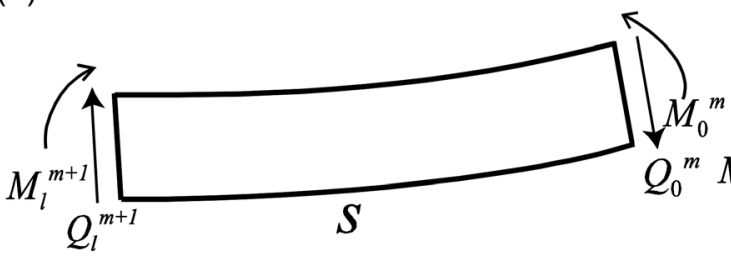

(b)

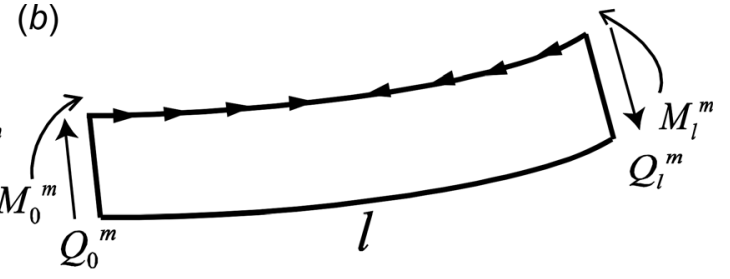

Fig. 3 Schematic of the $m$ th element consists of island and spacing: (a) free-body diagram of spacing between islands and $(b)$ free-body diagram of the $m$ th island 
Table 1 Geometrical and material parameters used in the simulations

\begin{tabular}{lcc}
\hline \hline Parameter & Definition & Value \\
\hline$E$ & Young's modulus & $20 \mathrm{GPa}$ \\
$L$ & Length of the beam & $22.2 \mathrm{~m}$ \\
$h$ & Height of the beam & $25 \mathrm{~mm}$ \\
$l$ & Length of the island & $0.1 \mathrm{~m}$ \\
$s$ & Separation between the islands & $0.1 \mathrm{~m}$ \\
$\tau$ & The maximum value of the traction & $100 \mathrm{kN} / \mathrm{m}$ \\
\hline \hline
\end{tabular}

$$
\begin{aligned}
Q_{l}^{m+1} & =\frac{\bar{\tau}}{6} \frac{M_{l}^{m}}{l}+\left(1-\frac{1}{12} \bar{\tau}\right) Q_{l}^{m}+\frac{\bar{\tau}}{30} \tau e \\
M_{l}^{m+1} & =\left(1-\frac{2-\rho}{12 \rho} \bar{\tau}\right) M_{l}^{m}-\left(\frac{1}{\rho}-\frac{5-3 \rho}{60 \rho} \bar{\tau}\right) Q_{l}^{m} l-\frac{(2-\rho) \bar{\tau}}{60 \rho} \tau e l
\end{aligned}
$$

where $\rho=(l / l+s)$ denotes the coverage ratio of the islands on the substrate.

For the island by the free end of the cantilever beam, we have $M_{l}{ }^{1}=Q_{l}{ }^{1}=0$, i.e., the initial condition.

We notice that when $(\bar{\tau} / 6 \rho) \ll 1$, Eq. (18) can be approximated as

$$
\begin{aligned}
& Q_{l}^{m+1}=\frac{M_{l}^{m}}{l} \sqrt{\frac{\bar{\tau} \rho}{6}} \sin \sqrt{\frac{\bar{\tau}}{6 \rho}}+Q_{l}^{m} \cos \sqrt{\frac{\bar{\tau}}{6 \rho}}+\frac{\tau e}{5} \sqrt{\frac{\bar{\tau} \rho}{6}} \sin \sqrt{\frac{\bar{\tau}}{6 \rho}} \\
& M_{l}^{m+1}=M_{l}^{m} \cos \sqrt{\frac{\bar{\tau}}{6 \rho}}-Q_{l}^{m} l \sin \sqrt{\frac{\bar{\tau}}{6 \rho}} / \sqrt{\frac{\bar{\tau} \rho}{6}}+\frac{\tau e l}{5}\left(\cos \sqrt{\frac{\bar{\tau}}{6 \rho}}-1\right)
\end{aligned}
$$

Thus, we have

$$
\begin{aligned}
& M_{l}^{m}=\frac{\tau e l}{5}\left(\cos \sqrt{\frac{\bar{\tau}}{6 \rho}}(m-1)-1\right) \\
& Q_{l}^{m}=\frac{\tau e}{5} \sqrt{\frac{\bar{\tau} \rho}{6}} \sin \sqrt{\frac{\bar{\tau}}{6 \rho}}(m-1)
\end{aligned}
$$

Substituting Eq. (20) into Eq. (15), the curvature of the $m$ th island can be simplified as

$$
k^{m}(x)=\frac{\tau e l}{E I}\left[\frac{1}{5}\left(\cos \sqrt{\frac{\bar{\tau}}{6 \rho}}(m-1+\rho(1-\bar{x}))-1\right)+\bar{x}(1-\bar{x})\right]
$$

With the bending moment and shear force in Eq. (20), we can obtain the curvature of the $m$ th spacing. On combining with Eq. (21), we are able to determine the curvature of an arbitrarily selected point in the beam. By integrating the curvature twice with respect to $x$, we then obtain the deflection of the beam. In the case that the number of islands $N$ is large, the overall curvature of the beam can be deduced from Eqs. (20) and (21) as

$$
\kappa=\left(\frac{\rho}{6}-\frac{1}{5}\right) \frac{\tau e l}{E I}
$$

Equation (22) connects the characteristics of the microscale islands with macroscopically measurable parameter. It could be used to determine the traction of adsorb islands (grains, cells, etc.) to the substrate.
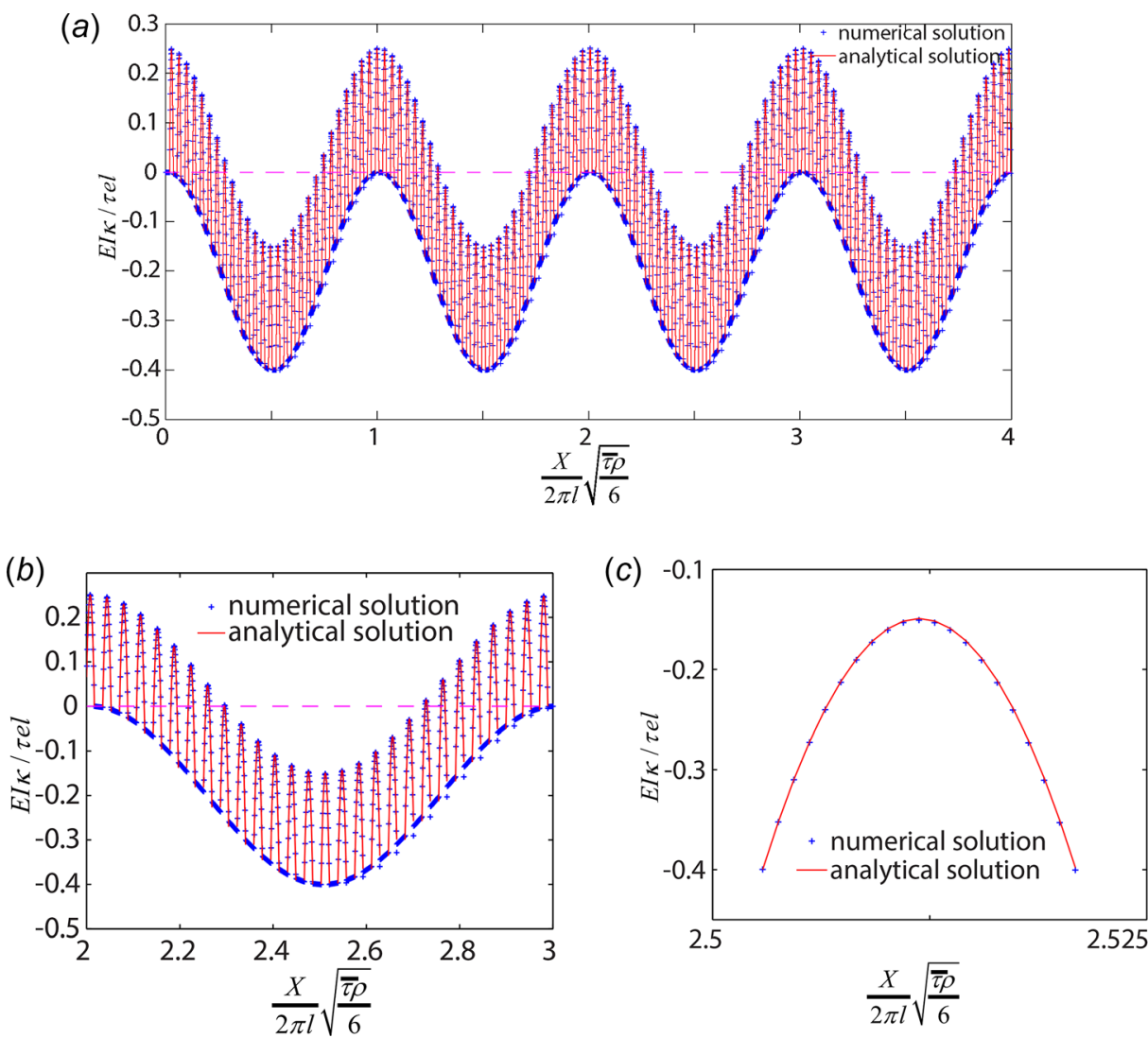

Fig. 4 The curvature of the beam with patterned islands, where $X$ denotes the coordinate from the fixed end of the beam (see Fig. 1): (a) curvature along the beam with 111 islands, (b) curvature within a period (with length $2 \pi I \sqrt{6 / \bar{\tau}} \rho)$ ), and (c) curvature within one island (with length I) 


\section{Numerical Verification and Analysis}

3.1 Comparison With Numerical Solution. To verify that the effect of the preceding approximation is small enough, we compare our analytical solutions with numerical solution which can be obtained by solving Eq. (6) numerically. We consider a cantilever beam as shown in Fig. 1. The geometry parameters and material parameters are listed in Table 1.

The results are graphed in nondimensional form $E I \kappa(X) / \tau e l$ versus $X / L$ in Fig. 4. The solid lines are the analytical results obtained from Eqs. (20) and (21), and the dots correspond to the numerical results. Clearly, the analytical results are exactly consistent with numerical results. Figure 4(a) shows that the curvature varies periodically along the longitudinal axis of the beam and the period equals to $2 \pi l \sqrt{6 / \bar{\tau} \rho}$ which can also be obtained from Eq. (21). The curvature distribution within a period and one island is shown in Figs. 4(b) and 4(c), respectively. From the perspective of whole length, the curvature curve is similar to cosine wave and within one island it is similar to para-curve which is mainly determined by the linear distribution of traction within the island. We can obtain from Fig. 4(a) that the maximum and minimum values of curvature are $\tau e l / 4 E I$ and $-2 \tau e l / 5 E I$, respectively.

In order to check the effects of the distribution of traction within islands on the curvature curve, we also consider the beam subjected to traction distribution within an island as $q(x)=16 \tau(x / l-1 / 2)^{3}$. By substituting this expression into Eq. (5), we obtain the governing equations for this case and solve the equations numerically. The curvature of the beam shows the same cosinelike distribution as shown in Fig. 4(a) although the amplitude of the curvature may change slightly. Regardless of the detailed distribution of traction within an island, the difference is very small as long as the resulted bending moment within an island is the same.

It is worth noting that the average curvatures of the beam shown in Fig. 4(a) are negative, which can also be obtained from Eq. (22). It gives an implication about the initial compressive stress measured during polycrystalline film deposition. Despite the distribution of the traction between islands and substrate while film deposition is far more different from linear distribution, it

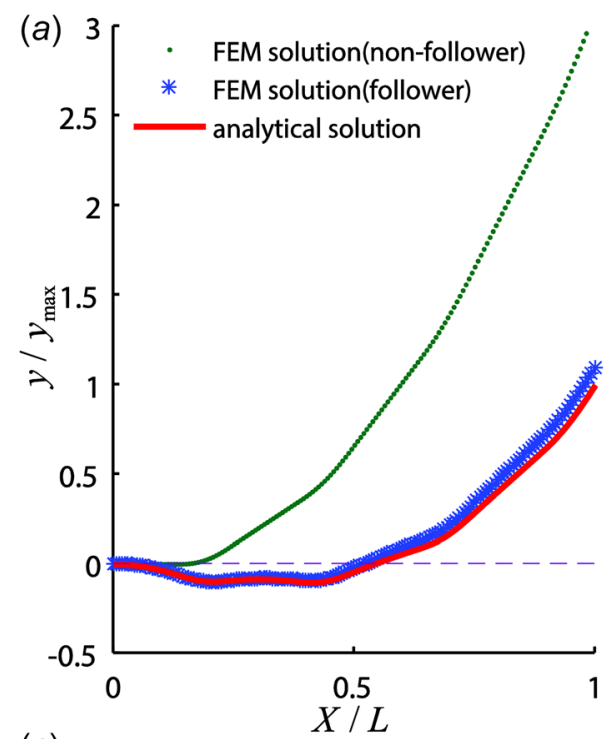

(c)

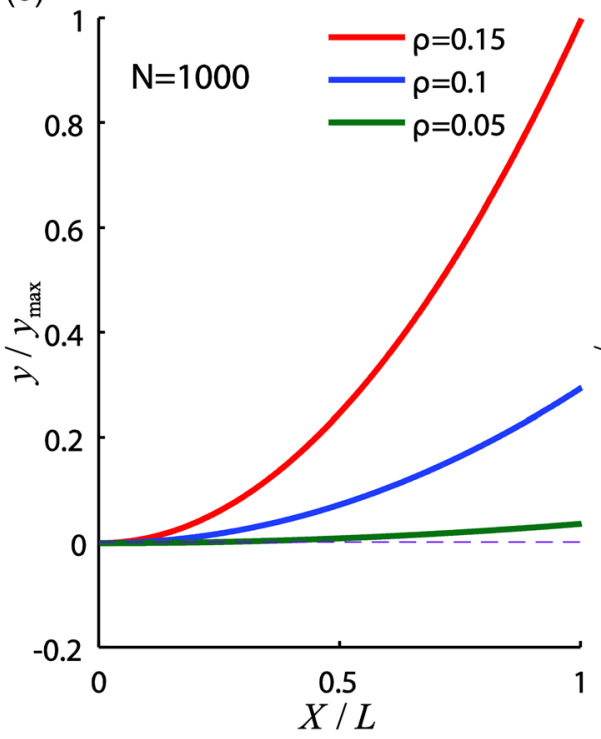

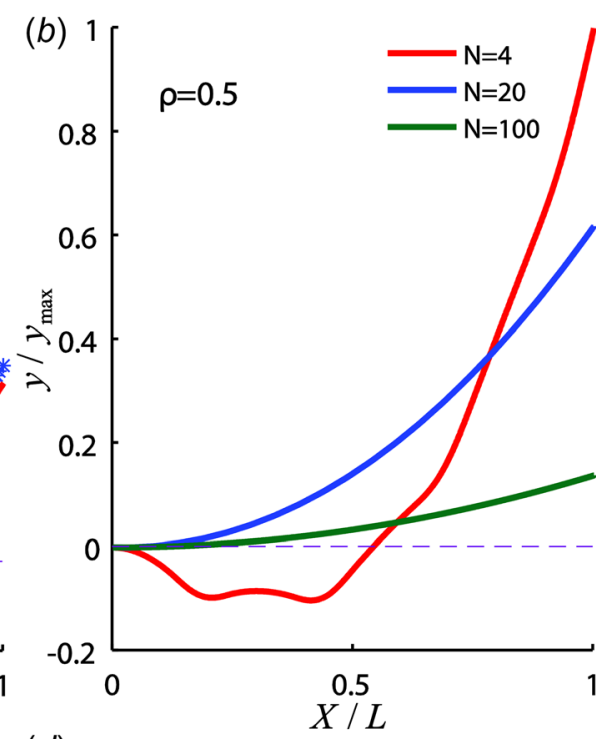

(d)

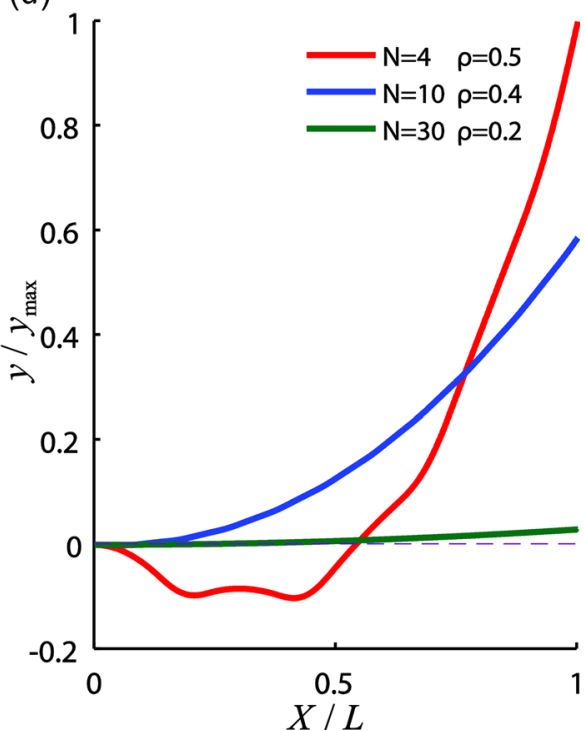

Fig. 5 Nondimensional deflection curve of the beam: (a) the solid line stands for analytical solution and dots are FEM results, $(b) \rho=0.5, N=4,20,100, y_{\max }$ corresponds to the maximal deflection of the beam when $\rho=0.5, N=4,(c) N=1000, \rho=0.05,0.1,0.15, y_{\max }$ is the maximal deflection of the beam when $\rho=0.15, N=1000$, and (d) $N$ decreases with increase in $\rho, y_{\max }$ refers to the maximal deflection of the beam when $\rho=0.5, N=4$ 
mainly affects the curvature distribution within the islands. During film deposition, the traction is concentrated near the island edge; hence, the curvature is basically invariant within the island [38]. When converting curvature to film stress using Stoney equation, the measured positive curvature is related to tensile stress and negative curvature implies compressive stress. Nevertheless, based on our analysis, the measured overall negative curvature can be induced by positive traction (i.e., tensile stress) introduced by an array of adsorbed islands. This can be explained as follows: the beam will bend when subjected to traction and the follower traction will induce a negative moment at the left-hand boundary of the island. This negative moment will be passed on to left neighboring island and gets accumulated. In the surface stress model proposed by Cammarata et al. [10], the entire island is in mechanical equilibrium. As a result of neglecting the effects between the islands, the measured negative curvature is interpreted to be induced by compressive stress. In addition, observing that our analysis begins from the island by the free end of the substrate, the curvature of the substrate with a few or one island can be inferred qualitatively from the right portion of Fig. 4(a). As the number of islands decreases as a result of island coalescence, the curvature becomes increasingly positive, which is in consistence with the experiment results $[8,39]$.

3.2 Theory Validation With Finite Element Method (FEM). We further perform finite element simulations to validate our analytical solutions. The parameters we investigated are given following the definition in Table 1 except that the length of the beam is reduced to $L=0.8 \mathrm{~m}$ in consideration of convergence.

When simulating with ABAQUS, we choose the three-dimensional solid element type C3D8I to investigate the bending of the beam. Note that in our model, the traction between adsorbed island and substrate is follower force which is always tangential to the surface. Therefore, large deformation model (NLGEOM) should be checked to enable follower traction. Besides, for comparison, we also simulate the case that the traction is nonfollower, i.e., the directions of traction remain horizontal.

We obtain the discrete deflection of the beam from ABAQUS simulation and analytical deflection curve by integrating the curvature obtained from Eqs. (20) and (21). The dimensionless deflection $y / y_{\max }$ versus $X / L$ diagram is plotted in Fig. 5(a), where $y_{\max }$ is the analytical value of the defection by the free end of the beam. As shown in Fig. 5(a), the analytical solution of deflection curve is in good agreement with the FEM results of the model subjected to follower traction and is much more less than the simulation results of nonfollower traction, which demonstrates the validity of our analytical solutions. In addition, we can see from Fig. 5(a) that for both FEM results of follower traction and our analytical results, the deflection of the beam near the fixed end is negative. This negative deflection is induced by a negative moment, which cannot be found for the case with nonfollower traction. Again, we show that follower traction indeed generates negative moment in the beam. While under the assumption of small deformation, the nonfollower traction is balanced within one island. Hence, in our model, follower traction cannot be simplified to nonfollower traction.

We also investigate the analytical deflection curve with different values of $N$ and $\rho$. The magnitude of the traction $\tau$ and beam properties remains unchanged. From Fig. 5(b), we see that the maximal deflection of the beam increases when the number of islands $N$ decreases and $\rho$ remains constant. The deflection of the beam is positively associated with the coverage ratio, when the number of island is substantial, see Fig. 5(c). It may correspond to the early stage of film deposition. We also consider the situation when $N$ decreases with increase in $\rho$ (see Fig. $5(d)$ ), a scenario of island coalescence. It may imply that the deflection increases with further deposition. In view of the magnitude of the traction increase with the length of the islands, this effect can be included by simply substituting the relationship into Eqs. (20) and (21).

\section{Conclusion}

Mechanical interactions between adsorbed islands and the substrate are regulated by the traction on the shared interface. This traction can induce stress in the islands and curvature in the substrate. We investigated an array of adsorbed islands sitting on a substrate beam and found out that the curvature varies periodically along the axis of the beam. The solution given here could complement with the currently broadly used Stoney equation as it is incapable of stress-measurement for films with adsorbed islands. Indeed, Haftbaradaran et al. [40] supplied a correction form of the Stoney equation for handling the stress introduced by patterned electrode island. In their treatment, the authors considered interfacial sliding due to excessive shear traction at the interface between islands and a cantilever beam. However, such a treatment, while moving one-step forward in contrast to the Stoney equation for stress evaluation in the film-patterned island system, still assumes that the traction is a nonfollower force which is in force and moment equilibrium within one island. Such scenario could not give rise to negative curvature prior to coalescence during polycrystalline film deposition. In reality, the traction is a follower force. We demonstrate that even positive traction, i.e., tensile stress, can also introduce negative curvature for follower tractions from the islands. Furthermore, we reveal that a simple relationship lies between the magnitude of traction force and the coverage ratio of the islands with the amplitude and period of the curvature, which can be used to develop a new strategy to measure cellular traction.

In short, we give the closed-form expression on the resulted curvature in substrate beams by distributed tractions. Such a relationship could be employed to monitor the stress evolution during thin film deposition, to quantify the stress level of cell traction as cells adhere to cantilever beams and other related mechanical systems like charging-discharging induced stress in island-patterned electrode films.

\section{Acknowledgment}

The authors acknowledge the support from the National Natural Science Foundation of China (NSFC) (Grant No. 11425211).

\section{References}

[1] Zhang, Z., and Lagally, M. G., 1997, "Atomistic Processes in the Early Stages of Thin-Film Growth," Science, 276(5311), pp. 377-383

[2] Thompson, C. V., and Carel, R., 1996, "Stress and Grain Growth in Thin Films," J. Mech. Phys. Solids, 44(5), pp. 657-673.

[3] Spaepen, F., 2000, "Interfaces and Stresses in Thin Films," Acta Mater., 48(1), pp. 31-42.

[4] Cammarata, R. C., 1994, "Surface and Interface Stress Effects in Thin Films," Prog. Surf. Sci., 46(1), pp. 1-38.

[5] Stoney, G. G., 1909, "The Tension of Metallic Films Deposited by Electrolysis," Proc. R. Soc. London Ser. A, 82(553), pp. 172-175.

[6] Freund, L. B., and Suresh, S., 2004, Thin Film Materials: Stress, Defect Formation and Surface Evolution, Cambridge University Press, Cambridge, UK.

[7] Chason, E., and Floro, J., 1996, "Measurements of Stress Evolution During Thin Film Deposition," MRS Proc., 428, p. 499.

[8] Floro, J. A., Chason, E., Cammarata, R. C., and Srolovitz, D. J., 2002, "Physical Origins of Intrinsic Stresses in Volmer-Weber Thin Films," MRS Bull., 27(1), pp. 19-25.

[9] Tello, J. S., and Bower, A. F., 2008, "Numerical Simulations of Stress Generation and Evolution in Volmer-Weber Thin Films," J. Mech. Phys. Solids, 56(8), pp. $2727-2747$.

[10] Cammarata, R., Trimble, T., and Srolovitz, D., 2000, "Surface Stress Model for Intrinsic Stresses in Thin Films," J. Mater. Res., 15(11), pp. 2468-2474.

[11] Rajamani, A., Sheldon, B. W., Chason, E., and Bower, A. F., 2002, "Intrinsic Tensile Stress and Grain Boundary Formation During Volmer-Weber Film Growth," Appl. Phys. Lett., 81(7), pp. 1204-1206.

[12] Tello, J. S., Bower, A. F., Chason, E., and Sheldon, B. W., 2007, "Kinetic Model of Stress Evolution During Coalescence and Growth of Polycrystalline Thin Films," Phys. Rev. Lett., 98(21), p. 216104.

[13] Sheldon, B. W., Bhandari, A., Bower, A. F., Raghavan, S., Weng, X., an Redwing, J. M., 2007, "Steady-State Tensile Stresses During the Growth of Polycrystalline Films," Acta Mater., 55(15), pp. 4973-4982.

[14] Chason, E., Shin, J., Chen, C.-H., Engwall, A., Miller, C., Hearne, S., and Freund, L., 2014, "Growth of Patterned Island Arrays to Identify Origins of Thin Film Stress,” J. Appl. Phys., 115(12), p. 123519. 
[15] Chason, E., Engwall, A., Miller, C., Chen, C.-H., Bhandari, A., Soni, S., Hearne, S., Freund, L., and Sheldon, B., 2015, "Stress Evolution During Growth of 1-D Island Arrays: Kinetics and Length Scaling," Scr. Mater., 97, pp. 33-36.

[16] Sethuraman, V. A., Chon, M. J., Shimshak, M., Srinivasan, V., and Guduru, P. R., 2010, "In Situ Measurements of Stress Evolution in Silicon Thin Films During Electrochemical Lithiation and Delithiation," J. Power Sources, 195(15), pp. 5062-5066.

[17] Pharr, M., Suo, Z., and Vlassak, J. J., 2013, "Measurements of the Fracture Energy of Lithiated Silicon Electrodes of Li-Ion Batteries," Nano Lett., 13(11), pp. 5570-5577.

[18] Bucci, G., Nadimpalli, S. P., Sethuraman, V. A., Bower, A. F., and Guduru, P. R., 2014, "Measurement and Modeling of the Mechanical and Electrochemical Response of Amorphous Si Thin Film Electrodes During Cyclic Lithiation," J. Mech. Phys. Solids, 62, pp. 276-294.

[19] Sethuraman, V. A., Chon, M. J., Shimshak, M., Van Winkle, N., and Guduru, P. R., 2010, "In Situ Measurement of Biaxial Modulus of Si Anode for Li-Ion Batteries," Electrochem. Commun., 12(11), pp. 1614-1617.

[20] Soni, S. K., Sheldon, B. W., Xiao, X., Verbrugge, M. W., Dongjoon, A., Haftbaradaran, H., and Huajian, G., 2011, "Stress Mitigation During the Lithiation of Patterned Amorphous Si Islands," J. Electrochem. Soc., 159(1), pp. A38-A43.

[21] Xiao, X., Liu, P., Verbrugge, M., Haftbaradaran, H., and Gao, H., 2011 , "Improved Cycling Stability of Silicon Thin Film Electrodes Through Patterning for High Energy Density Lithium Batteries," J. Power Sources, 196(3), pp. 1409-1416.

[22] Haftbaradaran, H., Xiao, X., Verbrugge, M. W., and Gao, H., 2012, "Method to Deduce the Critical Size for Interfacial Delamination of Patterned Electrode Structures and Application to Lithiation of Thin-Film Silicon Islands," J. Power Sources, 206, pp. 357-366.

[23] He, Y., Yu, X., Li, G., Wang, R., Li, H., Wang, Y., Gao, H., and Huang, X, 2012, "Shape Evolution of Patterned Amorphous and Polycrystalline Silicon Microarray Thin Film Electrodes Caused by Lithium Insertion and Extraction," J. Power Sources, 216, pp. 131-138.

[24] Kumar, R., Tokranov, A., Sheldon, B. W., Xiao, X., Huang, Z., Li, C., an Mueller, T., 2016, "In Situ and Operando Investigations of Failure Mechanisms of the Solid Electrolyte Interphase on Silicon Electrodes," ACS Energy Lett., 1(4), pp. 689-697.

[25] Discher, D. E., Janmey, P., and Wang, Y. L., 2005, "Tissue Cells Feel and Respond to the Stiffness of Their Substrate," Science, 310(5751), pp. 1139-1143.
[26] Kubow, K. E., Vukmirovic, R., Zhe, L., Klotzsch, E., Smith, M. L., Gourdon, D., Luna, S., and Vogel, V., 2015, "Mechanical Forces Regulate the Interactions of Fibronectin and Collagen I in Extracellular Matrix," Nat. Commun., 6, p. 8026.

[27] Harris, A. K., Stopak, D., and Wild, P., 1981, "Fibroblast Traction as a Mechanism for Collagen Morphogenesis," Nature, 290(5803), pp. 249-251.

[28] Rape, A. D., Guo, W. H., and Wang, Y. L., 2011, "The Regulation of Traction Force in Relation to Cell Shape and Focal Adhesions," Biomaterials, 32(8), pp. 2043-2051.

[29] Harris, A. K., Wild, P., and Stopak, D., 1980, "Silicone Rubber Substrata: New Wrinkle in the Study of Cell Locomotion," Science, 208(4440), pp. 177-179.

[30] Lee, J., Leonard, M., Oliver, T., Ishihara, A., and Jacobson, K., 1994, "Traction Forces Generated by Locomoting Keratocytes," J. Cell Biol., 127(6), pp. $1957-1964$.

[31] Dembo, M., and Wang, Y. L., 1999, "Stresses at the Cell-to-Substrate Interface During Locomotion of Fibroblasts," Biophys. J., 76(4), pp. 2307-2316.

[32] Tan, J. L., Tien, J., Pirone, D. M., Gray, D. S., Bhadriraju, K., and Chen, C. S. 2003, "Cells Lying on a Bed of Microneedles: An Approach to Isolate Mechanical Force," Proc. Natl. Acad. Sci. U.S.A., 100(4), pp. 1484-1489.

[33] He, S. J., Su, Y. W., Ji, B. H., and Gao, H. J., 2014, "Some Basic Questions on Mechanosensing in Cell-Substrate Interaction,” J. Mech. Phys. Solids, 70, pp. 116-135.

[34] Mertz, A. F., Banerjee, S., Che, Y., German, G. K., Xu, Y., Hyland, C., Marchetti, M. C., Horsley, V., and Dufresne, E. R., 2012, "Scaling of Traction Forces With the Size of Cohesive Cell Colonies," Phys. Rev. Lett., 108(19), p. 198101.

[35] Gardel, M. L., Sabass, B., Ji, L., Danuser, G., Schwarz, U. S., and Waterman, C. M., 2008, "Traction Stress in Focal Adhesions Correlates Biphasically With Actin Retrograde Flow Speed," J. Cell Biol., 183(6), pp. 999-1005.

[36] Timoshenko, S. P., and Goodier, J., 1970, Theory of Elasticity, McGraw Hill, New York.

[37] Braun, M., and Golubitsky, M., 1983, Differential Equations and Their Applications, Springer, New York.

[38] Thornton, J. A., and Hoffman, D., 1989, "Stress-Related Effects in Thin Films," Thin Solid Films, 171(1), pp. 5-31.

[39] Shull, A. L., and Spaepen, F., 1996, "Measurements of Stress During Vapor Deposition of Copper and Silver Thin Films and Multilayers," J. Appl. Phys., 80(11), pp. 6243-6256.

[40] Haftbaradaran, H., Soni, S. K., Sheldon, B. W., Xiao, X., and Gao, H., 2012 "Modified Stoney Equation for Patterned Thin Film Electrodes on Substrates in the Presence of Interfacial Sliding," ASME J. Appl. Mech., 79(3), p. 031018. 